\title{
Quantifying Self-Similarity in Cardiac Inter-Beat Interval Time Series
}

\author{
PE McSharry ${ }^{1,2}$, BD Malamud ${ }^{2,3}$ \\ ${ }^{1}$ Department of Engineering Science, University of Oxford, Oxford, UK \\ ${ }^{2}$ Mathematical Institute, University of Oxford, Oxford, UK \\ ${ }^{3}$ Department of Geography, King's College London, Strand, London, UK
}

\begin{abstract}
We compare and quantify the scaling and long-range persistence (long memory) in time series using five different techniques: power-spectral, wavelet variance, semivariograms, rescaled-range $(R / S)$ and detrended fluctuation analysis. We apply these techniques to both normal and log-normal synthetic fractional noises and motions generated using the spectral method, where a normally distributed white noise is appropriately filtered such that its power-spectral density, $S$, depends upon frequency, $f$, according to $S \sim f^{-\beta}$. Finally, we examine the longrange persistence of cardiac interbeat intervals. We find that for normal [N] and log-normal [LN] fractional noises: (1) power-spectral analysis does a reasonably good job at correctly quantifying the strength of long-range persistence for all $\beta[N]$ and $\beta>-0.5[L N]$; (2) semivariograms, $1.2<\beta<2.5$ [N and LN]; (3) rescaled range $0.0<\beta<0.8$ [N and LN]; (4) wavelet variance analysis all $\beta$ [N] and $\beta>-0.8$ [LN]; (5) detrended fluctuation analysis $-0.8<\beta<2.2$ [N] and $-0.2<\beta<2.2$ [LN].
\end{abstract}

\section{Introduction}

The human body relies on a number of control mechanisms to function efficiently. The complexity of the underlying physiological system gives rise to biomedical signals that often reflect non-stationary nonlinear dynamics. Biomedical signals recorded from healthy subjects typically display erratic fluctuations and contain long-range correlations. This contradicts the classical assumption of homeostasis where healthy systems are expected to selfregulate in an attempt to reduce variability [1].

The heart is particularly adaptable in terms of having the ability to quickly respond to external perturbations and changes in activity such as resting, walking, running and sleeping. This adaptability and underlying complexity of the heart may be quantified using an analysis of cardiac inter-beat time intervals derived from the electrocardiogram. Furthermore, this complexity appears to change in a characteristic way when the subjects age or experience disease, reflecting the subsequent loss of adaptability [2].

Self-affine time series, where the power-spectral density scales as a power of the frequency, appear in a wide variety of contexts; examples in biomedical engineering include cardiac rhythms and gait dynamics [1]. Stochastic time series are characterized by a statistical distribution of values and by their persistence. Persistence is the degree to which values in a time series are internally correlated and can be classified in terms of range, short or long, and strength, weak or strong. Self-affine time series are scale invariant, thus always exhibit long-range persistence. Here, we quantify synthetic self-affine time series with varying degrees of long-range persistence strength and put them into the context of of cardiac inter-beat intervals.

An analysis of the self-similarity of biomedical signals provides a means of distinguishing between health and disease and monitoring the effect of ageing [3,4]. This may offer the possibility of designing new diagnostic tools. The essential idea is to quantify the self-similarity of the recorded biomedical signal. One approach is to calculate the power-spectral density, $S(f)$, and estimate the powerlaw scaling exponent, $\beta$, that satisfies $S(f) \sim f^{-\beta}$. This exponent, $\beta$, may be viewed as a measure of the roughness of the time series, with smoother self-similar time series having larger values of $\beta$. The $1 / f$-like noise $(\beta=1)$ characteristic of cardiac inter-beat interval time series $[5,1]$ is midway between the complete randomness of white noise $(\beta=0)$ and the much smoother Brownian motion $(\beta=2)$.

\section{Methods}

In this section, five techniques are described that can be used to quantify self-similarity or persistence. Following [6], spectral analysis, semivariograms, rescaledrange and wavelet analysis for quantifying self-similarity are described. Furthermore, the notation $H_{a}, H_{u}, H_{w}$ is used to denote the Hausdorff exponent, Hurst exponent and wavelet variance exponent. This is done, following [6], as the traditional terminology of using $H$ for both the 
Hausdorff exponent and the Hurst exponent has resulted in considerable confusion in the literature. Another technique, known as detrended fluctuation analysis (DFA), is briefly summarised [7]. A method for generating synthetic data for the purpose of evaluating and comparing these five techniques is also described.

\subsection{Spectral analysis}

The Fourier transform, which is used to decompose the signal into sine and cosine basis functions, forms the basis of spectral analysis [8]. For a discrete time series $x_{n}$, $n=1, \ldots, N$, where the total duration of the signal, $T$, is divided into $N$ intervals of size $\delta=T / N$, the discrete Fourier transform (DFT) and inverse DFT are defined by

$$
X_{k}=\delta \sum_{n=1}^{N} x_{n} e^{2 \pi i n k / N}, \quad k=1, \ldots, N
$$

and

$$
x_{n}=\frac{1}{N \delta} \sum_{k=1}^{N} X_{k} e^{-2 \pi i n k / N}, \quad n=1, \ldots, N .
$$

The power-spectral density function $S_{k}$, is given by

$$
S_{k}=\lim _{N \rightarrow \infty} \frac{2\left|X_{k}\right|^{2}}{N \delta}, \quad k=1, \ldots, N / 2,
$$

where the factor of 2 in Eq. (3) arises because of summing up to $N / 2$ instead of $N$ as the Fourier coefficients, $X_{k}$, are symmetric, $\left|X_{k}\right|=\left|X_{N-k}\right|$. For a self-affine time series, $S_{k} \sim f_{k}^{-\beta}$ where $f_{k}=\frac{k}{N \delta}$ and $k=1, \ldots, N / 2$. In practice, the fast Fourier transform (FFT) provides a computationally efficient approach for determining the DFT [9]. A Welch window was employed to reduce spectral leakage. It is also important to remove the mean from the time series before windowing when estimating the power spectrum.

In terms of typical noise processes, white noise has approximately constant power densities, $S_{k}$, at all frequencies and has no correlations between values in the series giving $\beta=0$. In contrast, Brownian motion has strong long-range persistence with $\beta=2$. Cardiac inter-beat interval time series, for frequencies below $0.1 \mathrm{~Hz}$, have been described as a $1 / f$ noise process [5] or pink noise where the power-spectral analysis gives $\beta=1$.

\subsection{Semivariograms}

The semivariogram provides a method for measuring the long-range correlations of non-stationary time series [10]. For discrete time series, the semivariogram is given by

$$
\gamma_{k}=\frac{1}{2(N-k)} \sum_{n=1}^{N-k}\left(y_{n+k}-y_{n}\right)^{2},
$$

where $k$ is the lag. For a self-affine time series, the semivariogram scales as $\gamma_{k} \sim k^{2 H_{a}}$ where $H_{a}$ is the Hausdorff dimension.

\subsection{Rescaled-range}

Rescaled Range (R/S) analysis was first introduced by Hurst [11] in the context of his studies of the hydrology of the Nile river. Let the original time series have $N$ data points. For subsets of this time series with $k$ data points, $x_{n}, n=1, \ldots, k$, the mean, $\mu_{k}$ and standard deviation, $\sigma_{k}$, are calculated. The running sum, $y_{m}$, relative to the mean, $y_{m}=\sum_{n=1}^{m}\left(x_{n}-\mu_{k}\right)$ is then computed. Next, the range, $R_{k}=\max _{m} y_{m}-\min _{m} y_{m}$, dispersion, $S_{k}=\sigma_{N}$, and finally their ratio, $R_{k} / S_{k}$, are determined. Suppose the original time series has $N$ points. For successive subintervals, $k$ of $N$, the ratio, $R / S$ is computed. This may be achieved by considering $k=N, N / 2, N / 4, \ldots, 8$. For $k=N / q$, the data set is divided into $q$ parts and the quantity $R_{k} / S_{k}$ is computed $q$ times leading to an average $\left\langle R_{k} / S_{k}\right\rangle$. For a self-affine time series, $\left\langle R_{k} / S_{k}\right\rangle \sim\left(\frac{k}{2}\right)^{H_{u}}$, where $H_{u}$ is the Hurst exponent.

\subsection{Wavelet analysis}

The wavelet transform is a generalisation of the Fourier transform and provides information about both the spatial and frequency dependence of a time series [12]. It may be viewed as a filter $g\left[\left(t^{\prime}-t\right) / a\right]$ (centred at time $t$ with scale parameter $a$ ) that is convolved with a time series, $f(t)$,

$$
W(t, a)=\frac{1}{a^{1 / 2}} \int_{\infty}^{\infty} g\left(\frac{t^{\prime}-t}{a}\right) f\left(t^{\prime}\right) d t^{\prime} .
$$

The function $g(t)$ is known as the mother wavelet and is used to generate a family of rescaled wavelets with the normalisation factor $a^{1 / 2}$ ensuring that the transformed signal has the same energy at all scales. Furthermore, it is chosen to satisfy $\int_{-\infty}^{\infty} g(t) d t=0$. In the following, the Mexican hat wavelet, given by the second derivative of the Gaussian distribution,

$$
g\left(t^{\prime}\right)=\frac{1}{\sqrt{2 \pi}}\left(1-t^{\prime 2}\right) \exp \left(\frac{-t^{\prime 2}}{2}\right)
$$

is employed. For a self-affine time series (see [6]), the variance, $V_{W}$, of the wavelets $W(t, a)$ will scale with $a$ such that $V_{W} \sim a^{H_{W}}$.

\subsection{Detrended fluctuation analysis}

Detrended fluctuation analysis (DFA) was introduced for quantifying the self-similarity of nonstationary time series and is widely used for the analysis of biomedical signals [7]. The raw time series $x_{n}$ is first integrated to give 
$y_{n}=\sum_{i=1}^{n} x_{i}$ for $n=1, \ldots, N$. For each length scale, $m, y_{n}$ is divided into segments of equal length, $m$. In each segment, the data is detrended by subtracting the local linear least squares fit, $y_{k}^{(m)}$. The root-mean-square fluctuation of this integrated and detrended time series is given by

$$
F(m)=\sqrt{\frac{1}{N} \sum_{k=1}^{N}\left[y_{k}-y_{k}^{(m)}\right]^{2}} .
$$

The average fluctuation, $F(m)$, is then calculated for each segment $m$. For a self-affine time series, $F(m)$ scales with $m$ according to $F(m) \sim m^{\alpha}$.

\subsection{Synthetic data}

Normally distributed white noise is an example of a stationary process with $\beta=0$. Brownian motion, obtained by summing a white noise time series, is a non-stationary process with $\beta=2$. We distinguish fractional noises, $\beta<1$, from fractional motions, $\beta>1$ in an attempt to separate weak long-range persistence, $0<\beta<1$, from strong long-range persistence, $\beta>1$. Those fractional noises with $\beta<0$ are considered "anti-persistence". In general, any self-affine time series with power-spectral density exponent $\beta$ can be transformed to $\beta^{\prime}=\beta+2$ by summation and $\beta^{\prime}=\beta-2$ by differentiation.

A realisation of noise processes with different values of $\beta$ were obtained by (i) computing the DFT of a normally distributed white noise, (ii) filtering the resulting Fourier coefficients, $X_{k}$, using $X_{k}^{\prime}=(k / N)^{-\beta / 2} X_{k}$, and (iii) taking the inverse DFT of the filtered Fourier coefficients, $X_{k}^{\prime}$.

A total of 25 synthetic time series with normal distributions were produced, each with 4,096 points. In order to assess the robustness of each of the five techniques for dealing with non-normally distributed time series, which is often the case for cardiac inter-beat intervals, we also used log-normal distributions. The coefficient of variation, $\zeta=\sigma / \mu$, defined as the ratio of the standard deviation, $\sigma$, to the mean, $\mu$, provides a description of these distributions. Along with the normally distributed synthetic time series, we also considered coefficients of variation of $\zeta=0.25$ and $\zeta=0.5$.

\section{Results}

The five techniques described in Section 2 were used to estimate the scaling exponents of time series with normal distributions and log-normal distributions with coefficients of variation of $\zeta=0.25$ and $\zeta=0.5$. Figure 1 summarises the results of each technique applied to our synthetic time series. The $x$-axis represents the "benchmark" synthetic fractional noises with the known distributions (normal and
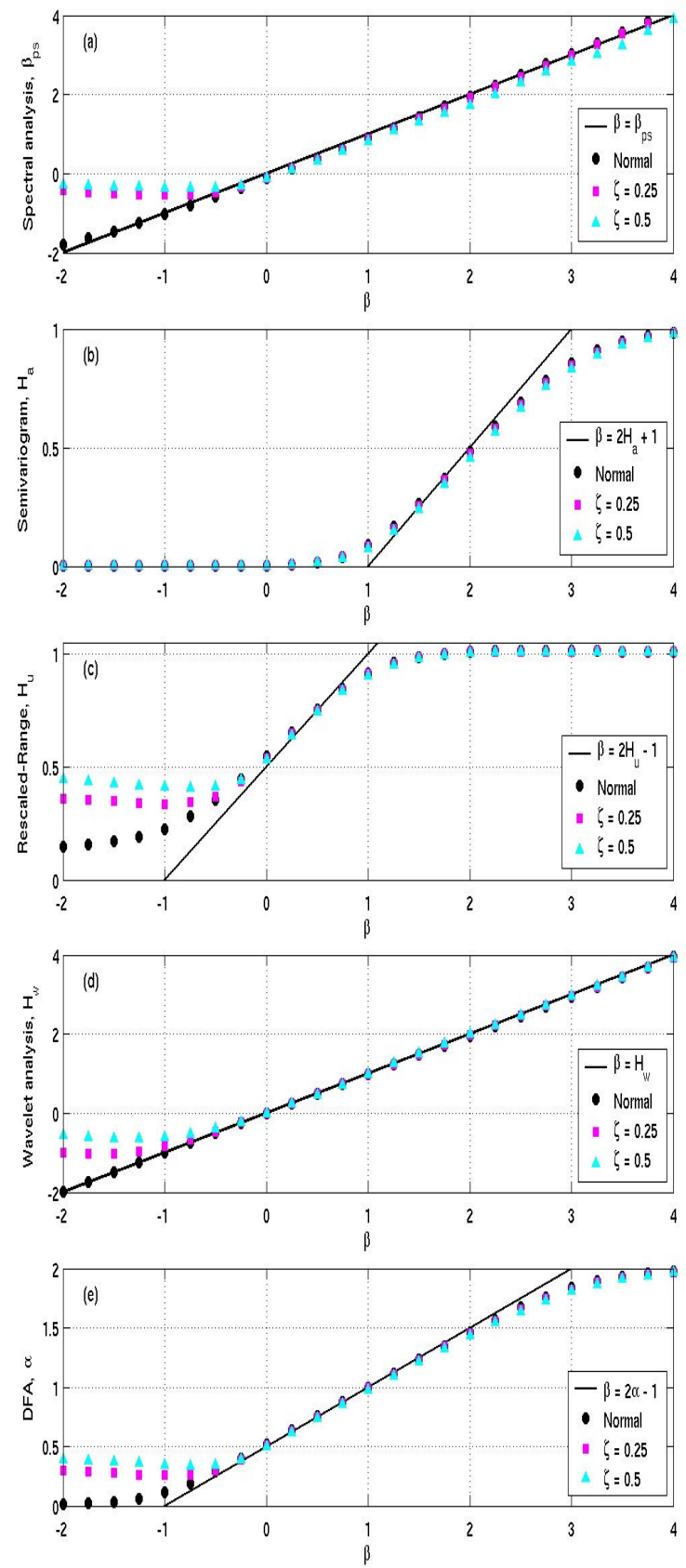

Figure 1. Scaling exponent estimates using: (a) spectral analysis; (b) semivariograms; (c) rescaled-range analysis; (d) wavelet analysis; and (e) detrended fluctuation analysis (DFA). 
log-normal) and different strengths of long-range persistence ranging over $-2 \leq \beta \leq 4$. The $y$-axis shows the estimated exponents using each technique as a function of the theoretical values of $\beta$. We now describe the relationships between the different exponents obtained from these five techniques and the theoretical exponent $\beta$.

Spectral analysis: The power-spectral density exponent, $\beta=\beta_{p s}$, quantifies self-similarity for almost all values of $\beta$ with normally distributed time series. For log-normal fractional noises, the results are biased for $\beta<-0.5$.

Semivariogram: The semivariogram provides an estimate of the Hausdorff exponent, $H_{a}$, satisfying $\beta=2 H_{a}+1$ in the range $1.2<\beta<2.5$ for both normal and log-normal fractional noises.

Rescaled-range: Rescaled-range provides an estimate of the Hurst exponent, $H_{u}$, satisfying $\beta=2 H_{u}-1$ for $0<\beta<0.8$ with accuracy very poor for $\beta<0$.

Wavelet analysis: The wavelet analysis provides an estimate of $H_{w}$, which satisfies $\beta=H_{W}$ for the entire range $-2 \leq \beta \leq 4$. For $\beta<-0.8$ the estimates are poor for non-normal noises.

Detrended fluctuation analysis: DFA gives $\beta=2 \alpha-1$ for $-0.8<\beta<2.2$ for both normal and log-normal noises. For log-normal noises, accuracy is reasonable for $-0.2<\beta<2.2$.

Finally we used the MIT-BIH Normal Sinus Rhythm Database, available from Physionet [13], to investigate the 24-hour cardiac rhythms of a group of subjects with no significant arrhythmias, including 5 men (aged 26 to 45) and 13 women (aged 20 to 50). These time series contained between 74 and 114 thousand data points. We employed the most accurate and robust of the five techniques considered here, wavelet analysis, to estimate $\beta$ and found that $\beta=1.13 \pm 0.19$ (mean \pm standard deviation).

\section{Conclusion}

This analysis has shown that the techniques available for estimating the scaling of self-similar time series provide varying levels of accuracy depending on $\beta$ and the degree of non-normality of the time series being considered. For normally distributed time series, semivariograms provide accurate estimates for $1.2<\beta<2.5$, rescaled-range for $0.0<\beta<0.8$, DFA for $-0.8<\beta<2.2$, and powerspectra and wavelets for all values of $\beta$. All techniques demonstrate decreasing accuracy for log-normal fractional noises with increasing coefficient of variance, particularly for antipersistent time series.

Wavelet analysis offers the best performance both in terms of providing accurate estimates for normally distributed time series over the entire range $-2 \leq \beta \leq 4$ and having the least decrease in accuracy for log-normal noises. The range of values of $\beta$ estimated for a group of healthy subjects, $\beta=1.13 \pm 0.19$, confirms that cardiac rhythms are similar to pink noise $(\beta=1)$. Furthermore the analysis suggests that spectral, wavelet and detrended fluctuation analysis are the most suitable techniques for investigating cardiac inter-beat interval time series.

\section{References}

[1] Goldberger AL, Amaral LAN, Hausdorff JM, Ivanov PC, Peng CK, Stanley HE. Fractal dynamics in physiology: alterations with disease and ageing. Proc Natl Acad Sci 2002; 99:2466-2472.

[2] Ivanov PC, Amaral LAN, Goldberger AL, Havlin S, Rosenblum MG, Struzik ZR, Stanley HE. Multifractality in human heartbeat dynamics. Nature 1999;399:461-465.

[3] Amaral LAN, Goldberger AL, Ivanov PC, Stanley HE. Scale-independent measures and pathologic cardiac dynamics. Phys Rev Lett 1998;81(11):2388-2391.

[4] Peng CK, Hausdorff JM, Goldberger AL. Fractal mechanisms in neural control: Human heartbeat and gait dynamics in health and disease. In Nonlinear Dynamics, SelfOrganization, and Biomedicine. Cambridge, UK: Cambridge University Press, 2000; 66-96.

[5] Kobayashi M, Musha T. 1/f fluctuation of heartbeat period. IEEE Trans Biomed Eng 1982;29:456.

[6] Malamud BD, Turcotte DL. Self-affine time series: I. generation and analyses. Advances in Geophysics 1999;40:1-90.

[7] Peng CK, Havlin S, Stanley HE, Goldberger AL. Quantification of scaling exponents and crossover phenomena in nonstationary heartbeat time series. Chaos 1995;5:82-87.

[8] Priestly MB. Spectral Analysis and Time Series. London: Academic Press, 1981.

[9] Press WH, Flannery BP, Teukolsky SA, Vetterling WT. Numerical Recipes in C. 2nd edition. Cambridge: CUP, 1992.

[10] Mandelbrot BB, Van Ness JW. Fractional brownian motions, fractional noises and applications. SIAM review 1968;10:422-437.

[11] Hurst HE. Long-term storage capacity of reservoirs. Trans Am Soc Civil Engineers 1951;116:770-808.

[12] Grossman A, Morlet J. Decomposition of hardy function into square integrable wavelets of constant shape. SIAM J Math Anal 1984;15:723-736.

[13] Goldberger AL, Amaral LAN, Glass L, Hausdorff JM, Ivanov PC, Mark RG, Mietus JE, Moody GB, Peng CK, Stanley HE. Physiobank, physiotoolkit, and physionet: Components of a new research resource for complex physiologic signals. Circulations 2000;101(23):e215-e220. URL www. physionet.org.

Address for correspondence:

Patrick E. McSharry

Dept. of Engineering Science / University of Oxford

Parks Road/ Oxford OX1 3PJ / UK

tel./fax: +44-1865-273095/273905

patrick@mcsharry.net 